\title{
Human Macrophages Utilize the Podosome Formin FMNL1 for Adhesion and Migration
}

\author{
Matthew R. Miller, Scott D. Blystone* \\ Department of Cell \& Developmental Biology, SUNY Upstate Medical University, New York, USA \\ Email: blystons@upstate.edu
}

Received 14 February 2015; accepted 1 March 2015; published 4 March 2015

Copyright (C) 2015 by authors and Scientific Research Publishing Inc.

This work is licensed under the Creative Commons Attribution International License (CC BY). http://creativecommons.org/licenses/by/4.0/

(c) (i) Open Access

\section{Abstract}

Macrophages play a crucial role in detecting, regulating, and resolving immune crises, requiring migration through complex extracellular matrices. Unwarranted macrophage inflammatory activity potentiates kidney disease, rheumatoid arthritis, and transplant rejection. Proper remodeling of the actin cytoskeleton, especially at adhesion structures, is essential to the translocation of macrophages. Macrophages form actin-rich adhesions termed "podosomes", giving them the capacity to make contacts with the substratum for traction through interstitial tissues. Macrophages express multiple formins, including FMNL1, Dia1, and Fhod1, with potential to impact actin remodeling involved in migration. Formins are a family of proteins that are best known for modifying the actin cytoskeleton via nucleation, elongation, bundling, and/or severing actin filaments. In this study we demonstrate that the formin FMNL1 is a key regulator of podosomes and is required for normal macrophage migration. Additionally, this is the first study to demonstrate defects in primary human cell migration resulting from specific formin silencing. Pharmacologic inhibition of all formin activity results in a significant decrease in podosome formation and normal macrophage migration. Furthermore, targeted suppression of FMNL1 results in decreases in macrophage migration similar to inhibition of all expressed macrophage formins. These novel findings suggest FMNL1 as a possible chemotherapeutic target to hinder macrophage migration, which could offer an innovative method for limiting unnecessary macrophage-mediated inflammation. We hypothesize that formins are required in podosome actin dynamics to support macrophage migration.

\section{Keywords}

FRL1, Monocyte, Extravasation, Chemotaxis

\footnotetext{
${ }^{*}$ Corresponding author.
} 


\section{Introduction}

Macrophages form actin-dense adhesion structures termed "podosomes". These contain a dense pillar-like actin core that is tethered to the cortical actin cytoskeleton with radial actin filaments [1]. Recent proteomic analysis of macrophage podosomes has identified over 200 proteins, including signaling, linking, and actin-associated proteins [2]. Podosomes were first described as small membrane domains resembling macrophage feet [3]. These structures are enriched with multiple types of integrins, making intricate contacts with the extracellular matrix and mediating adhesion [4] [5]. Numerous integrin-associated proteins alter the dynamics of podosomes. With a rapid turnover rate between 2 and 12 minutes, podosomes form either de novo or through fission of preexisting podosomes while terminating their lifespan via fusion or disintegration [6]. These adhesion structures have also been implicated in migration, as their absence has been observed to lead to defects in chemokine-induced migration [5] [8]. Furthermore, podosomes have been shown to be present in migrating macrophages in both a 2D and 3D environment, indicating their importance for adhesion and migration [9] [10]. Podosomes have also been shown to be directly associated with matrix degradation and remodeling through release of metalloproteinases [11]. An accumulation of research strongly implicates that podosomes are critical for macrophage migration; however the signaling pathways and functional mechanisms associated with these structures requires further study.

Formins are actin-binding proteins that have the capacity for nucleating, polymerizing, capping, severing and/ or bundling actin [12]-[14]. These proteins perform these functions in homodimeric form, through conserved formin homology 2 domains (FH2) [15]. There are 15 known mammalian formins, which are categorized into 7 different groups by domain and phylogenetic relationships [16]. Substantial effort has begun to define the functions of these proteins in vitro; however the study of the cellular function of formins remains limited. Formins have been implicated in variety of cellular processes including cell division, adhesion, migration, vesicular transport, polarity, and signaling [17]-[21].

Previous studies in our lab have identified that the formin FMNL1 (formin-like 1) is associated with macrophage podosomes and is localized to the apex of the pillar-shaped dense actin core [22]. FMNL1 was first isolated from mRNA in murine brain and spleen samples [23]. Two separate isoforms were identified, $(\alpha$ and $\beta)$, and were hypothesized to play an important role in macrophage function [23]. Interestingly, FMNL1 has been observed to have the ability to perform actin filament nucleation, polymerization, bundling and severing in vitro [24]-[26]. To date, FMNL1 has been observed to have three isoforms, $(\alpha, \beta$, and $\gamma)$, but specific functions of these splice variants have not been ascribed [23] [27]. Other studies have suggested FMNL1 is important for the structural integrity of the golgi complex and also for membrane localization for induction of blebbing [27] [28].

We have previously reported that FMNL1 is associated with macrophage podosomes [22]. FMNL1 was shown to be necessary for podosome stability. siRNA-mediated suppression of FMNL1 resulted in a decrease in podosome number, abnormal podosomal actin dynamics, and a subsequent loss of macrophage adhesion. In these studies, it was not determined whether FMNL1 had any role in macrophage migration or whether other formins contributed to macrophage adhesion or podosome dynamics. Since it is imperative for macrophages to make podosomes to migrate through the extracellular matrix, these exciting observations lead us to test whether FMNL1 is necessary for macrophage migration. In this study, we have determined that FMNL1 plays a pivotal role in the migration of human peripheral blood monocyte-derived macrophages.

\section{Materials \& Methods}

Except were noted, all culture reagents were from Invitrogen (Carlsbad, CA, USA) and all plasticware from Corning Incorporated (Corning, NY, USA).

\subsection{Macrophage Isolation and Differentiation}

Peripheral blood was drawn into a $60 \mathrm{ml}$ syringe containing $7.5 \mathrm{ml}$ of 6\% Dextran (Pharmacosmos, Holbaek, Denmark) in HBSS $^{-}$for red blood cell sedimentation, and $5.625 \mathrm{ml}$ of $96 \mathrm{mM}$ sodium citrate (Sigma-Aldrich, St. Louis, MO, US) in $\mathrm{HBSS}^{-}$as an anti-coagulant. The blood was then transferred to $50 \mathrm{ml}$ conical tubes for red blood cell sedimentation for 30 minutes at room temperature. Plasma and white blood cells were removed and placed on top of Ficoll-Paque PLUS (Amersham Pharmacia Biotech AB, Uppsala, Sweden) in a $50 \mathrm{ml}$ conical tube and centrifuged at $3000 \mathrm{~g}$ at $4^{\circ} \mathrm{C}$ for 30 minutes. The buffy coat layer was removed and washed in $\mathrm{HBSS}^{-}$ 
at $1000 \mathrm{~g}$ at $4^{\circ} \mathrm{C}$ for 10 minutes. Monocyte pellets were resuspended in complete media (RPMI-1640 containing 20\% FBS (Gemini Bio-Products, West Sacremento, CA, USA), $2 \mathrm{mM}$ glutamax, $50 \mu \mathrm{g} / \mathrm{ml}$ gentamicin (Sigma-Aldrich, St. Louis, MO, USA)), and induced to differentiate with $125 \mathrm{pg} / \mathrm{ml}$ GM-CSF (Berlex, Seattle, WA, USA). Monocytes were cultured in complete media and allowed to differentiate into macrophages at $37^{\circ} \mathrm{C}$ and $5 \% \mathrm{CO}_{2}$. Macrophages were used between day 5 and day 10 of culture.

\subsection{Formin Inhibition and Adhesion Assays with SMIFH2}

Adherent macrophages were washed quickly using ice cold $10 \mathrm{mM}$ EDTA (Sigma-Aldrich, St. Louis, MO, USA) in $\mathrm{HBSS}^{-}$, and then $5 \mathrm{ml}$ of ice cold $10 \mathrm{mM}$ EDTA in $\mathrm{HBSS}^{-}$was added to the flask and placed at $4^{\circ} \mathrm{C}$ for approximately 10 minutes. The bottom of the flask was smacked to help break adhesion of macrophages, and macrophages were gently detached from the flask using a disposable cell scraper. Macrophages were removed from flask and transferred to a $50 \mathrm{ml}$ conical tube and washed twice with complete media. Macrophages were resuspended in $5 \mathrm{ml}$ complete media and counted using a hemocytometer (Reichert, Buffalo, NY, USA). Macrophages were plated in 24 well plates containing glass coverslips (Fisher Scientific, Pittsburg, PA, USA) at a density of 120,000 cells/ well. Macrophages were allowed to adhere to the glass coverslips overnight. Small molecule inhibitor of the FH2 domain (SMIFH2) (Sigma-Aldrich, St. Louis, MO, USA) inhibitor was diluted into multiple concentrations in complete media, including $5 \mu \mathrm{M}, 10 \mu \mathrm{M}, 20 \mu \mathrm{M}, 30 \mu \mathrm{M}$, and DMSO as a vehicle control (Sigma-Aldrich, St. Louis, MO, USA). Media was aspirated from wells containing macrophages and replaced with regular complete media and treatment medias in duplicate at 10 minute intervals to allow counting at each treatment condition. Adhered macrophages were counted at the center of each coverslip using a $20 \times$ objective at $0,15,30,60$, and 120 minutes for each treatment. Student's t-test was performed for statistical analysis.

To determine viability, in parallel samples, media was aspirated from wells containing macrophages and replaced with regular complete media and treatment medias. Plates were placed back in incubator for 16 hours, the timespan used for migration assays. After the 16 hour time point, media was collected for each treatment and adhered macrophages were lifted and collected as previously described and added to collected media. Macrophage viability was performed for each treatment using trypan exclusion (Sigma-Aldrich, St. Louis, MO, USA).

\subsection{Podosome Analysis}

Macrophages were prepared as previously described. SMIFH2 inhibitor was diluted in complete media to a concentration of $30 \mu \mathrm{M}$ with DMSO as a vehicle control. Media was aspirated from wells containing macrophages and replaced with regular complete media and treatment medias. Plates were placed back in incubator for two hours. Macrophages were then fixed using 3.7\% ice cold formaldehyde (Fisher Scientific, Fair Lawn, NJ, USA) for 1 hour at $4^{\circ} \mathrm{C}$ followed by permeabilization using ice cold 0.002\% NP-40 (BMD Biosciences Inc, La Jolla, CA, USA) in PBS for 10 seconds. Macrophages were then treated with blocking buffer in PBS containing 0.01\% Triton X-100 (Fisher Scientific, Pittsburg, PA, USA) and 3\% goat serum (Invitrogen, Carlsbad, CA, USA) in PBS for 30 minutes at room temperature. Macrophages were stained with rhodamine phalloidin (Cytoskeleton, Denver, CO, USA) at [1:10,000] in PBS for 30 minutes, washed with PBS five times, and the coverslips were then mounted on glass slides (Globe Scientific, Paramus, NJ, USA) using an anti-fading solution. Using a Nikon Eclipse E800 fluorescent microscope (Nikon, Melville, NY, USA) equipped with a Hamamatsu ORCA-ER digital camera (Bridgewater, NJ, USA) with NIS-Elements software, macrophages were imaged, analyzed, and quantified for either exhibiting at least five podosomes per cell (actin-dense structures with a diameter of approximately $0.5 \mu \mathrm{m}$ as described by Mersich et al. 2010, or having less than five podosomes per cell. Student's t-test was performed for statistical analysis.

\subsection{Macrophage Migration}

Macrophages were prepared as previously described. Macrophages were removed from flasks and transferred to a $50 \mathrm{ml}$ conical tube and washed twice with RPMI with gentamicin. Macrophages were resuspended in $1 \mathrm{ml}$ RPMI with gentamicin and counted using a hemocytometer. Transwell permeable polycarbonate support inserts with $5.0 \mu \mathrm{m}$ pores were placed into wells of a 24 well plate. $600 \mu \mathrm{l}$ of complete media was added into the lower chamber. The upper chamber was loaded with 175,000 macrophages resuspended in RPMI with gentamicin and 
either SMIFH2 inhibitor, DMSO, or nothing to a final volume of $100 \mu$. The migrations assays were placed in the incubator for 16 hours. After which, the media was removed from the upper well of the inserts and the membranes were fixed in $3.7 \%$ formaldehyde in PBS at $4^{\circ} \mathrm{C}$ for 1 hour. The inserts were allowed to air dry for 1 hour. Polycarbonate membranes were stained using a Diff-Quick staining kit (IMEB Inc., San Marcos, CA, USA) following the manufacturer's protocol. The inserts were then placed upside down and allowed to dry overnight. Membranes were cut from the transwell inserts and placed upside down on glass slides, covered with a drop of immersion oil (Cargille Laboratories, Cedar Grove, NJ, USA), and covered with a coverslip. Microscopy on the membranes was performed using light microscopy with a $40 \times$ objective and five different fields-ofview were counted for each membrane. Student's t-test was performed for statistical analysis.

\section{5. siRNA Knockdown of FMNL1 in Macrophages}

Macrophages were transduced with small interference RNA molecules targeting FMNL1 (Ambion, Inc, Austin, TX, USA) using the INTERFERin transduction reagent (PolyPlus Transfection, New York, NY, USA). Three unique target sequences were used for FMNL1 along with a scramble oligo for control. Macrophages prepared as above following 5 - 10 days of differentiation were seeded 1 day in advance of siRNA transduction in T25 flasks at $\sim 2.0 \times 10^{6}$ macrophages in $5 \mathrm{ml}$ complete media. On the day of transduction, complexes of siRNA and INTERFERin were prepared in $400 \mathrm{ul}$ of serum-free media following the manufacturer's protocol. A concentration of $600 \mathrm{nM}$ siRNA in $4 \mathrm{ml}$ of media was used to replace the media in the T25 flask. Macrophages were cultured with siRNA for 72 hours and harvested as described above for Western blot analysis or migration assays as previously described. For Western blot analysis, $150 \mu \mathrm{g}$ of each macrophage lysate was prepared with sample buffer, loaded into a $10 \%$ SDS polyacrylamide gel, and resolved using electrophoresis. Separated proteins were transferred from the gel to a PVDF membrane (Millipore, Billerica, MA, USA), blocked with 3\% BSA (Lampire Biological Laboratories, Pipersville, PA, USA) in TBS (Fisher Scientific, Fair Lawn, NJ, USA) buffer, and then probed for FMNL1 using mouse monoclonal 2369E4a (Santa Cruz, CA, USA) and transaldolase goat polyclonal T-20 (Santa Cruz, CA, USA) for a loading control. Student's t-test was performed for statistical analysis.

\section{Results}

\subsection{Global Inhibition of Formins Suppresses Macrophage Migration}

We have previously reported that FMNL1 was required for normal podosome dynamics in macrophages [22]. siRNA-mediated depletion of FMNL1 decreased macrophage adhesion. Several formins have been ascribed roles in the migration of various cell types. To determine whether either FMNL1 or other formins affect macrophage migration, we first assessed migration following treatment with SMIFH2, a general formin inhibitor. Studies have shown SMIFH2 to have a concentration-dependent effect on formin mediated assembly of filamentous actin with a half-maximum inhibition at approximately $15 \mu \mathrm{M}$ [29]. When compared with non-treated macrophages, there was a $9.1 \%$ and $34.7 \%$ decrease in the number of macrophages that were observed to migrate across a barrier when treated with $20 \mu \mathrm{M}$ and $30 \mu \mathrm{M}$ SMIFH2 respectively, as seen in Figure 1. These results imply that at the reported working concentration of SMIFH2, normal macrophage migration is disrupted when formin function is inhibited. Interestingly, although there is a substantial decrease in migration at $30 \mu \mathrm{M}$, treatment does not completely inhibit migration.

\subsection{FMNL1 Contributes to Macrophage Migration}

Previous studies in our lab have shown that the formin FMNL1 plays an important role in macrophage adhesion and podosome stability [22]. As pharmacologic inhibition of all macrophage formins suppressed migration, we directly assessed the contribution of FMNL1 to formin activity in macrophage migration. Human macrophages were treated separately with 3 different FMNL1 targeting oligos or scrambled siRNA for 72 hours for Western blot and migration analysis in parallel. Using a modified Boyden chamber, siRNA treated macrophages were subjected to the migration assay and cells that moved through the porous barrier were quantified as described in Methods. The targeting oligos si2226, si2227, and si2228 showed a $70.7 \%, 57.7 \%$, and $65.8 \%$ reduction in FMNL1 protein expression, respectively, as compared to transaldolase loading control as shown in representative Western blots in Figure 2(b). This decrease in FMNL1 caused a 46\%, 39.3\%, and 41.1\% reduction in macrophage migration, respectively, as shown in Figure 2(a). The similarity in decreased macrophage migration 


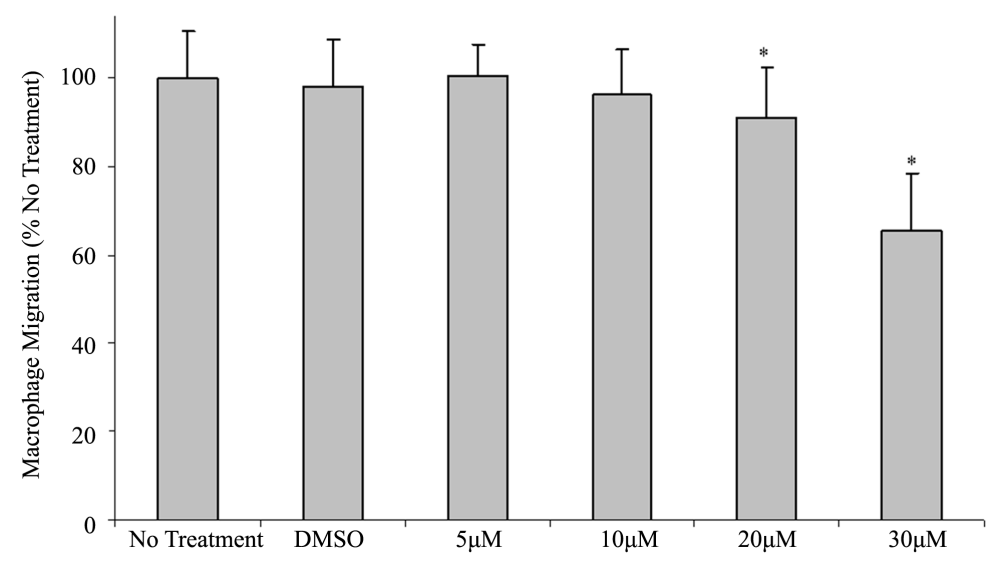

Figure 1. SMIFH2 effects on macrophage migration. Human macrophages were treated with SMIFH2 and challenged to cross a porous barrier as described in Methods. Macrophages that migrated through the barrier were quantified using light microscopy after performing differential staining. Shown are averages with (+/-) standard deviation of the mean of four experiments performed in duplicate with five cell counts per sample. Asterisks between groups indicates a $\mathrm{p}<0.05$.

(a)

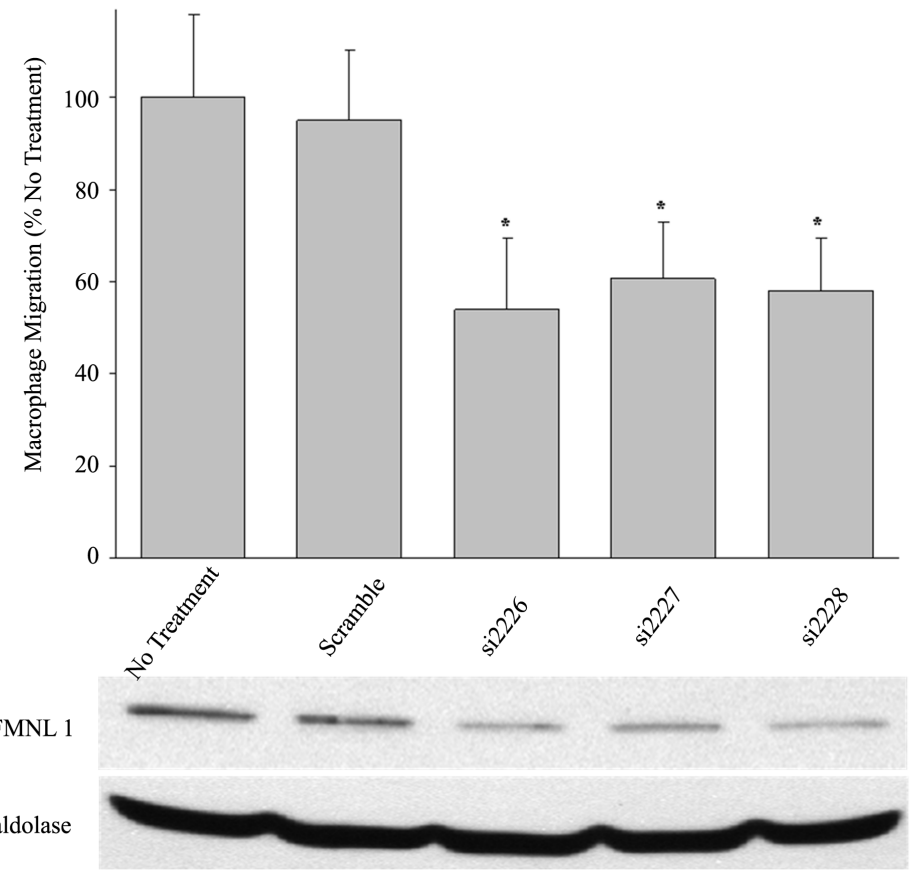

Figure 2. siRNA targeted deletion of FMNL1 significantly reduces macrophage migration. (a) Human peripheral blood monocyte derived macrophages were challenged to migrate across a porous barrier using a modified Boydenchamber in tandem following siRNA mediated reduction of FMNL1 as described in Methods. Macrophages that migrated through the barrier were quantified using light microscopy after performing differential staining. Shown are averages with (+/-) standard deviation of the mean of four experiments with five macrophage counts per sample. Asterisks indicates $\mathrm{p}<0.05$; (b) Western blot analysis of FMNL1 protein levels in macrophage lysates performed in parallel to migration analysis using transaldolase as a loading control. The Western blot is a representative of three experiments with equivalent outcomes. 
following inhibition of all formins (Figure 1) and specific suppression of FMNL1 (Figure 2(a)) suggests that FMNL1 is the formin exhibiting the largest role in macrophage migration.

\subsection{SMIFH2 Suppresses Macrophage Podosomes}

Human macrophages were plated on glass coverslips at identical densities and treated with $30 \mu \mathrm{M}$ SMIFH 2 or DMSO. After 2 hours of inhibitor treatment, macrophages were fixed, permeabilized, and stained with rhodamine phalloidin for actin visualization to quantitate podosomes. Podosomes were identified by staining for associated proteins including talin, vinculin, and paxillin as previously described [22] (data not shown). Using fluorescent microscopy, macrophages exhibiting podosomes were quantitated as described in Methods. Treatment with SMIFH2 at concentrations of $5 \mu \mathrm{M}$ caused a significant decrease in the numbers of macrophages displaying podosomes as shown in Figure 3(a). At concentrations above $5 \mu \mathrm{M}$, podosome number decreased at each consecutive timepoint from 15 minutes to 2 hours (data not shown). Further, at $30 \mu \mathrm{M}$, SMIFH2 caused remaining adhered macrophages to exhibit few to zero podosomes as observed in Figure 3(b). These observations correlate well with our previous demonstrations that siRNA mediated knockdown of FMNL1 suppresses podosome number in macrophages and in macrophage cell lines. Additionally, these results aid in explaining the loss of adhesion in SMIFH2 treated cells due to the significant loss of podosomes. It should be noted that these data underestimate the SMIFH2 effects on podosome numbers as cells eventually detach and are lost from analysis as significant podosome losses are incurred.

\subsection{SMIFH2 Induces Macrophage Detachment}

To determine if inhibition of formin function using SMIFH2 affects the stabilization of macrophage adhesion, a dose and time dependent assay was performed. In this study, we replaced media in wells containing glass coverslip-adhered macrophages at identical density with media containing varying concentrations of SMIFH2. Ma

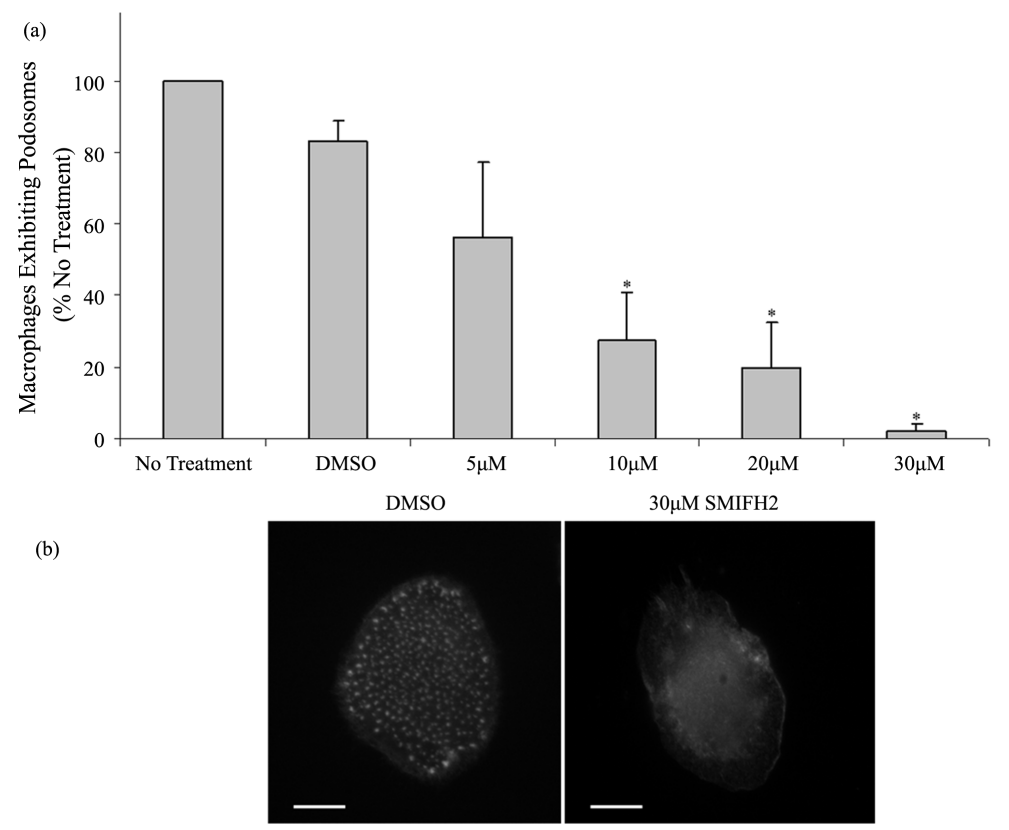

Figure 3. SMIFH2 effects on macrophage podosomes. (a) Human macrophages were treated with multiple concentrations of SMIFH2 and podosomes were counted as described in Methods. Shown are averages with (+/-) standard deviation of the mean of four experiments performed in duplicate with 100 cell counts per sample. Asterisks indicates $\mathrm{p}<0.05$; (b) Macrophages were stained with rhodamine phalloidin for actin visualization following 30 $\mu \mathrm{M}$ SMIFH2 as described in Methods. These images are representative of six experiments performed in duplicate with cell analysis of 100 cells per each sample. Scale bar is $10 \mu \mathrm{m}$. 
crophage de-adhesion was assessed after 15, 30, 60, and 120 minutes and in a dose dependent manner by SMIFH2. We determined that SMIFH2 at $30 \mu \mathrm{M}$ reduced adherent macrophages by $>50 \%$ within 120 minutes of treatment with no effect on cell viability, as shown in Figure 4. Therefore, we determined that the previously reported working concentration of SMIFH2 was the same for primary human macrophages. Our studies suggest that inhibition of formins induces macrophage de-adhesion. This is consistent with a model where continual podosome dynamics are dependent on formins such as FMNL1 and required for adhesion and likely migration.

\section{Discussion}

Macrophages are crucial for appropriate immune responses to combat the invasion of pathogens and promoting wound healing. For macrophages to reach sites of inflammation and tissue damage, they must have the capacity to firmly adhere and migrate through complex extracellular matrices. This macrophage targeting mechanism is reliant on the proper formation and functioning of podosomes. Studies performed on Wiskott-Aldrich syndrome (WASP) models have reported that the lack of the protein WASp causes a loss of podosome formation [30]. These macrophages were observed to still exhibit random motility, however directed migration was largely diminished [8]. Additionally, studies on dendritic cells from chronic myeloid leukemia (CML) patients showed absence of podosomes and defects in cell migration [7]. These studies support for a requirement for podosomes in resolving immune perturbations, and highlight questions of how these adhesion structures are created, regulated, and utilized for macrophage migration. In the studies discussed in this manuscript, we propose that the formin FMNL1 is important for podosome stability and is also a key component of the macrophage migration machinery.

Previous studies in our lab have indicated that FMNL1 is an indispensible protein for normal macrophage podosome dynamics. These findings were obtained via knockdown of FMNL1 protein expression and analysis of cell adhesion using light microscopy [22]. After these studies were performed, an inhibitor of formins, SMIFH2, was identified, characterized, and made commercially available. This inhibitor was described to halt the function of formins through targeting the actin binding motif within the formin homology 2 domain (FH2), preventing formins from nucleating, elongating, and attaching to the barbed end of actin filaments [29]. SMIFH2 has also been utilized in studies suggesting that formins play an important role in actin filaments that are generated for protrusive forces in interstitial migration of neutrophils [31]. The availability of SMIFH2 has allowed us to determine whether formins play a role in macrophage migration.

We demonstrated that SMIFH2 treatment of macrophages significantly reduces their macrophages capacity to migrate across a porous barrier. These data indicate that formins play a critical role in cellular changes during migration. However, this observation could result from an array of formin associated events including actin cytoskeleton remodeling, signaling, or even adapter protein functions. It remains unclear which actin regulating

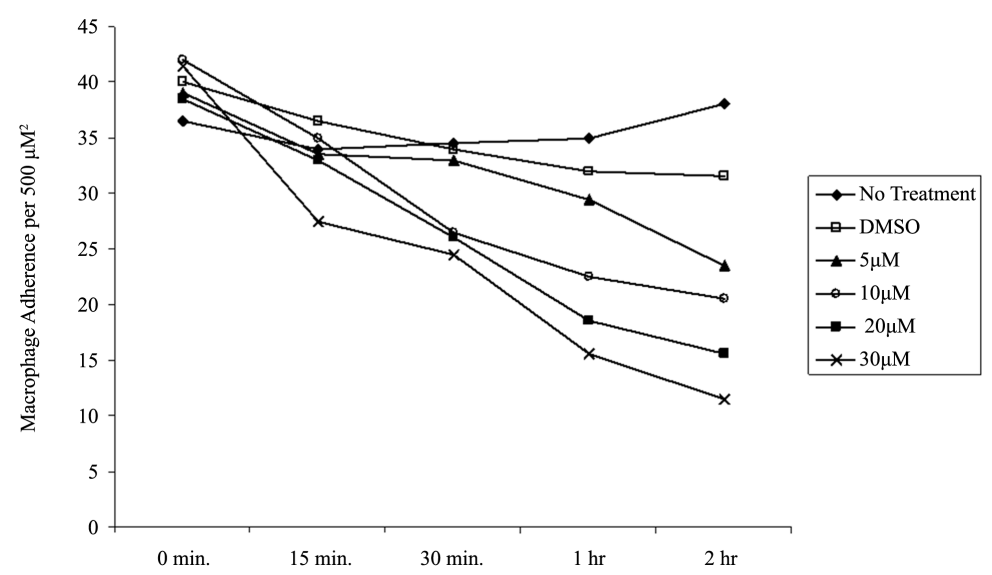

Figure 4. SMIFH2 induces macrophage detachment. Human macrophages were treated with multiple concentrations of SMIFH2 and attached macrophages remaining at each time point were quantified by visual counting as described in Methods. The graph is a representative of six experiments performed in duplicate with similar outcomes. 
events most formins may be performing in different cell types at any given time. Another interesting aspect of this data is that macrophage migration was not inhibited completely. This suggests some aspects of macrophage migration use podosomes, but additional mechanisms must exist, because all podosomes are lost by formin inhibition with $30 \mu \mathrm{M}$ SMIFH2, but only $50 \%$ of macrophage migration is inhibited. This may mean that formins are important for optimal migration, or alternatively, formins may be important for a certain mode of migration such as mesenchymal or amoeboid [32] [33]. Forcing cells into a single mode of migration may retard efficiency and there may be variation in mechanisms used between different modes of migration. Macrophages have been observed to use two modes of migration in heterogeneous environments, where mesenchymal migration is employed for dense tissue and amoeboid migration for less compact tissue [34]. Furthermore, studies have demonstrated that macrophages lose their capacity for mesenchymal migration coincident with loss of podosomes, but amoeboid migration seems to be unperturbed [11] [34] [35]. Our observation is not without its own caveat though, as SMIFH2 inhibits all formins, making it hard to distinguish which macrophage formin or formins is/ are contributing to migration. Nonetheless, this exciting data reveals that formins do indeed have an important role in a macrophage's ability to migrate normally. Alternatively, the partial inhibitory effects of SMIFH2 may suggest a dual role for formins wherein one is dependent on actin binding and inhibited by SMIFH2 while another is independent of actin binding and unaffected by SMIFH2.

We have previously reported that multiple formins, including Dia1, Dia3, FHOD1, FMNL2, and FMNL1, are expressed at high mRNA levels in human macrophages [36]. We have performed Western blot analysis on human macrophage lysates indicating high protein levels of multiple formins (data not shown). However, little is known about most of the functions of these formins in macrophages. Dia1 and FMNL1 have been implicated in phagocytosis and we have shown FMNL1 is critical for adhesion, but the other formins have yet to be characterized for their function and localization [22] [37] [38]. Since we have shown FMNL1 to be vital to macrophage adhesion and podosome stability, it seems likely to also be important for migration. It has yet to be determined whether the function of any specific formin directly impacts macrophage migration. Studies have shown that stationary and polarized macrophages form podosomes in 2D analysis and that macrophages form podosomes in 3D environments during mesenchymal migration [30] [39]. Additionally, as previously mentioned, studies on macrophages that have no WASp are incapable of directed migration, and do not form podosomes [8]. We have shown that the loss of FMNL1 expression in macrophages causes loss of podosomes [22]. Therefore, we examined the effects on macrophage migration when FMNL1 expression is reduced.

In our study we observed a significant decrease in macrophage migration across a porous barrier when FMNL1 protein levels were knocked down via siRNA. This observation ties together our previous observations that reduction of FMNL1 expression causes a loss in podosome stability and formation. Another interesting observation was that the reduction in macrophage migration using the formin inhibitor at $30 \mu \mathrm{M}$ was $34.6 \%$, and the average of the three siRNA targeting oligos was 42.1\%. This suggests that FMNL1 is more than likely the largest contributing formin to macrophage migration. Since we have shown FMNL1 to localize to podosomes, and when it is knocked down podosomes are lost, we have further verified the necessity not only for FMNL1 in macrophage migration, but also for normal podosome dynamics. In accordance with our previous studies using siRNA knockdown of formins, we also observed that pharmacological manipulation of formins similarly effects podosome stability and adhesion in macrophages. It was interesting to observe that macrophage adhesion and podosome stability were affected by formin inhibition and siRNA-mediated knockdown of FMNL1 similarly. This is the first study to demonstrate changes in primary human cell migration caused by specific silencing of an individual formin. These exciting findings delineate the necessity of formins in macrophage migration and highlight FMNL1 as an important formin in this process.

As this research has shed some light on the requirement of formins in migration, many other questions remain to be investigated. It is still unclear how FMNL1 is recruited to macrophage podosomes. It has been suggested that spatial and temporal regulation of FMNL1 is controlled by small Rho GTPases, including Rac1, and CDC42 [23] [40]. This would theoretically lead to the effector proteins of GTPases, guanine nucleotide exchange factors (GEFs) and GTPase activating proteins (GAPs), being the regulators of formin activation and localization. How these proteins are up or down-regulated at podosomes remains an active area of research. Another interesting, yet unknown event is the exact actin-associated mechanism(s) that FMNL1 is performing at the macrophage podosome. Due to limited technology to observe these functions in live cells and because FMNL1 has been observed to exhibit every formin attribute in vitro, it remains difficult to definitively determine the mechanism of FMNL1 at podosomes. As previously mentioned, podosomes are dynamic structures that turn 
over rapidly and during their lifespan both grow in size and then dissolve [6]. It seems probable that FMNL1 is critical in the maturation and stabilization of podosomes at their peak level of adhesion as we have demonstrated FMNL1 loss causes significantly reduced podosome numbers with corresponding loss of adhesion. Therefore, we hypothesize FMNL1 is either polymerizing de novo actin filaments and possibly being the driving force of the center actin pillar, or FMNL1 is bundling existing actin filaments in the core to maintain podosome structure during the adhesion lifespan. We do not yet have sufficient information to determine if FMNL1, or any other formin, is limited to one or many different functions in cellular environments.

Our observations support FMNL1 as a target for chemotherapeutic intervention to inhibit macrophage migration. The work presented in this paper strongly suggests that the formin FMNL1 plays a prominent role in this immune process. Further studies on FMNL1 in this process may pave the way to understanding and attenuating macrophage mediated diseases such as atherosclerosis and rheumatoid arthritis.

\section{Acknowledgements}

This work was supported by NIH DK79884 to S.D.B.

\section{Conflict of Interest Disclosure}

The authors declare no conflict of interest.

\section{References}

[1] Luxenburg, C., Geblinger, D., Klein, E., Anderson, K., Hanein, D., Geiger, B. and Addadi, L. (2007) The Architecture of the Adhesive Apparatus of Cultured Osteoclasts: From Podosome Formation to Sealing Zone Assembly. PLoS One, 2, e179. http://dx.doi.org/10.1371/journal.pone.0000179

[2] Cervero, P., Himmel, M., Kruger, M. and Linder, S. (2012) Proteomic Analysis of Podosome Fractions from Macrophages Reveals Similarities to Spreading Initiation Centres. European Journal of Cell Biology, 91, 908-922. http://dx.doi.org/10.1016/j.ejcb.2012.05.005

[3] Davies, W.A. and Stossel, T.P. (1977) Peripheral Hyaline Blebs (Podosomes) of Macrophages. Journal of Cell Biology, 75, 941-955. http://dx.doi.org/10.1083/jcb.75.3.941

[4] Marchisio, P.C., Bergui, L., Corbascio, G. C., Cremona, O., D’Urso, N., Schena, M., Tesio, L. and Caligaris-Cappio, F. (1988) Vinculin, Talin, and Integrins Are Localized at Specific Adhesion Sites of Malignant B Lymphocytes. Blood, 72, 830-833.

[5] Duong, L.T. and Rodan, G.A. (2000) PYK2 Is an Adhesion Kinase in Macrophages, Localized in Podosomes and Activated by Beta(2)-Integrin Ligation. Cell Motility and the Cytoskeleton, 47, 174-188. http://dx.doi.org/10.1002/1097-0169(200011)47:3<174::AID-CM2>3.0.CO;2-N

[6] Evans, J.G., Correia, I., Krasavina, O., Watson, N. and Matsudaira, P. (2003) Macrophage Podosomes Assemble at the Leading Lamella by Growth and Fragmentation. Journal of Cell Biology, 161, 697-705.

http://dx.doi.org/10.1083/jcb.200212037

[7] Dong, R., Cwynarski, K., Entwistle, A., Marelli-Berg, F., Dazzi, F., Simpson, E., Goldman, J.M., Melo, J.V., Lechler, R.I., Bellantuono, I., Ridley, A. and Lombardi, G. (2003) Dendritic Cells from CML Patients Have Altered Actin Organization, Reduced Antigen Processing, and Impaired Migration. Blood, 101, 3560-3567. http://dx.doi.org/10.1182/blood-2002-06-1841

[8] Jones, G.E., Zicha, D., Dunn, G.A., Blundell, M. and Thrasher, A. (2002) Restoration of Podosomes and Chemotaxis in Wiskott-Aldrich Syndrome Macrophages Following Induced Expression of WASp. The International Journal of Biochemistry \& Cell Biology, 34, 806-815. http://dx.doi.org/10.1016/S1357-2725(01)00162-5

[9] Carman, C.V., Sage, P.T., Sciuto, T.E., de la Fuente, M.A., Geha, R.S., Ochs, H.D., Dvorak, H.F., Dvorak, A.M. and Springer, T.A. (2007) Transcellular Diapedesis Is Initiated by Invasive Podosomes. Immunity, 26, 784-797. http://dx.doi.org/10.1016/j.immuni.2007.04.015

[10] Cougoule, C., Van Goethem, E., Le Cabec, V., Lafouresse, F., Dupre, L., Mehraj, V., Mege, J.L., Lastrucci, C. and Maridonneau-Parini, I. (2012) Blood Leukocytes and Macrophages of Various Phenotypes Have Distinct Abilities to form Podosomes and to Migrate in 3D Environments. European Journal of Cell Biology, 91, 938-949. http://dx.doi.org/10.1016/j.ejcb.2012.07.002

[11] Cougoule, C., Le Cabec, V., Poincloux, R., Al Saati, T., Mege, J.L., Tabouret, G., Lowell, C.A., Laviolette-Malirat, N. and Maridonneau-Parini, I. (2010) Three-Dimensional Migration of Macrophages Requires Hck for Podosome Organization and Extracellular Matrix Proteolysis. Blood, 115, 1444-1452. http://dx.doi.org/10.1182/blood-2009-04-218735 
[12] Pruyne, D., Evangelista, M., Yang, C., Bi, E., Zigmond, S., Bretscher, A. and Boone, C. (2002) Role of Formins in Actin Assembly: Nucleation and Barbed-End Association. Science, 297, 612-615. http://dx.doi.org/10.1126/science.1072309

[13] Wallar, B.J. and Alberts, A.S. (2003) The Formins: Active Scaffolds That Remodel the Cytoskeleton. Trends in Cell Biology, 13, 435-446. http://dx.doi.org/10.1016/S0962-8924(03)00153-3

[14] Goode, B.L. and Eck, M.J. (2007) Mechanism and Function of Formins in the Control of Actin Assembly. Annual Review of Biochemistry, 76, 593-627. http://dx.doi.org/10.1146/annurev.biochem.75.103004.142647

[15] Copeland, J.W., Copeland, S.J. and Treisman, R. (2004) Homo-Oligomerization Is Essential for Factin Assembly by the Formin Family FH2 Domain. The Journal of Biological Chemistry, 279, 50250-50256. http://dx.doi.org/10.1074/jbc.M404429200

[16] Higgs, H.N. and Peterson, K.J. (2005) Phylogenetic Analysis of the Formin Homology 2 Domain. Molecular Biology of the Cell, 16, 1-13. http://dx.doi.org/10.1091/mbc.E04-07-0565

[17] Kato, T., Watanabe, N., Morishima, Y., Fujita, A., Ishizaki, T. and Narumiya, S. (2001) Localization of a Mammalian Homolog of Diaphanous, mDia1, to the Mitotic Spindle in HeLa Cells. Journal of Cell Science, 114, 775-784.

[18] Bretscher, A. (2003) Polarized Growth and Organelle Segregation in Yeast: The Tracks, Motors, and Receptors. The Journal of Cell Biology, 160, 811-816. http://dx.doi.org/10.1083/jcb.200301035

[19] Yamana, N., Arakawa, Y., Nishino, T., Kurokawa, K., Tanji, M., Itoh, R.E., Monypenny, J., Ishizaki, T., Bito, H., Nozaki, K., Hashimoto, N., Matsuda, M. and Narumiya, S. (2006) The Rho-mDia1 Pathway Regulates Cell Polarity and Focal Adhesion Turnover in Migrating Cells through Mobilizing Apc and c-Src. Molecular and Cellular Biology, 26, 6844-6858. http://dx.doi.org/10.1128/MCB.00283-06

[20] Gupton, S.L., Eisenmann, K., Alberts, A.S. and Waterman-Storer, C.M. (2007) mDia2 Regulates Actin and Focal Adhesion Dynamics and Organization in the Lamella for Efficient Epithelial Cell Migration. Journal of Cell Science, 120, 3475-3487. http://dx.doi.org/10.1242/jcs.006049

[21] Young, K.G. and Copeland, J.W. (2010) Formins in Cell Signaling. Biochimica et Biophysica Acta, 1803, $183-190$. http://dx.doi.org/10.1016/j.bbamcr.2008.09.017

[22] Mersich, A.T., Miller, M.R., Chkourko, H. and Blystone, S.D. (2010) The Formin FRL1 (FMNL1) Is an Essential Component of Macrophage Podosomes. Cytoskeleton, 67, 573-585. http://dx.doi.org/10.1002/cm.20468

[23] Yayoshi-Yamamoto, S., Taniuchi, I. and Watanabe, T. (2000) FRL, a Novel Formin-Related Protein, Binds to Rac and Regulates Cell Motility and Survival of Macrophages. Molecular and Cellular Biology, 20, 6872-6881. http://dx.doi.org/10.1128/MCB.20.18.6872-6881.2000

[24] Harris, E.S., Li, F. and Higgs, H.N. (2004) The Mouse Formin, FRLalpha, Slows Actin Filament Barbed End Elongation, Competes with Capping Protein, Accelerates Polymerization from Monomers, and Severs Filaments. The Journal of Biological Chemistry, 279, 20076-20087. http://dx.doi.org/10.1074/jbc.M312718200

[25] Harris, E.S., Rouiller, I., Hanein, D. and Higgs, H.N. (2006) Mechanistic Differences in Actin Bundling Activity of Two Mammalian Formins, FRL1 and mDia2. The Journal of Biological Chemistry, 281, 14383-14392. http://dx.doi.org/10.1074/jbc.M510923200

[26] Esue, O., Harris, E.S., Higgs, H.N. and Wirtz, D. (2008) The Filamentous Actin Cross-Linking/Bundling Activity of Mammalian Formins. Journal of Molecular Biology, 384, 324-334. http://dx.doi.org/10.1016/j.jmb.2008.09.043

[27] Han, Y., Eppinger, E., Schuster, I.G., Weigand, L.U., Liang, X., Kremmer, E., Peschel, C. and Krackhardt, A.M. (2009) Formin-Like 1 (FMNL1) Is Regulated by N-Terminal Myristoylation and Induces Polarized Membrane Blebbing. The Journal of Biological Chemistry, 284, 33409-33417. http://dx.doi.org/10.1074/jbc.M109.060699

[28] Colon-Franco, J.M., Gomez, T.S. and Billadeau, D.D. (2011) Dynamic Remodeling of the Actin Cytoskeleton by FMNL1gamma Is Required for Structural Maintenance of the Golgi Complex. Journal of Cell Science, 124, 3118-3126. http://dx.doi.org/10.1242/jcs.083725

[29] Rizvi, S.A., Neidt, E.M., Cui, J., Feiger, Z., Skau, C.T., Gardel, M.L., Kozmin, S.A. and Kovar, D.R. (2009) Identification and Characterization of a Small Molecule Inhibitor of Formin-Mediated Actin Assembly. Chemistry Biology, 16, 1158-1168. http://dx.doi.org/10.1016/j.chembiol.2009.10.006

[30] Linder, S., Nelson, D., Weiss, M. and Aepfelbacher, M. (1999) Wiskott-Aldrich Syndrome Protein Regulates Podosomes in Primary Human Macrophages. Proceedings of the National Academy of Sciences of the United States of America, 96, 9648-9653. http://dx.doi.org/10.1073/pnas.96.17.9648

[31] Wilson, K., Lewalle, A., Fritzsche, M., Thorogate, R., Duke, T. and Charras, G. (2013) Mechanisms of Leading Edge Protrusion in Interstitial Migration. Nature Communications, 4, 2896. http://dx.doi.org/10.1038/ncomms3896

[32] Pankova, K., Rosel, D., Novotny, M. and Brabek, J. (2010) The Molecular Mechanisms of Transition between Mesenchymal and Amoeboid Invasiveness in Tumor Cells. Cellular and Molecular Life Sciences, 67, 63-71. 
http://dx.doi.org/10.1007/s00018-009-0132-1

[33] Le Cabec, V., Van Goethem, E., Guiet, R. and Maridonneau-Parini, I. (2011) Phagocyte Migration: An Overview. Médecine/Sciences, 27, 1112-1120. http://dx.doi.org/10.1051/medsci/20112712018

[34] Van Goethem, E., Poincloux, R., Gauffre, F., Maridonneau-Parini, I. and Le Cabec, V. (2010) Matrix Architecture Dictates Three-Dimensional Migration Modes of Human Macrophages: Differential Involvement of Proteases and Podosome-Like Structures. The Journal of Immunology, 184, 1049-1061. http://dx.doi.org/10.4049/jimmunol.0902223

[35] Maridonneau-Parini, I. (2014) Control of Macrophage 3D Migration: A Therapeutic Challenge to Limit Tissue Infiltration. Immunological Reviews, 262, 216-231. http://dx.doi.org/10.1111/imr.12214

[36] Krainer, E.C., Ouderkirk, J.L., Miller, E.W., Miller, M.R., Mersich, A.T. and Blystone, S.D. (2013) The Multiplicity of Human Formins: Expression Patterns in Cells and Tissues. Cytoskeleton, 70, 424-438. http://dx.doi.org/10.1002/cm.21113

[37] Brandt, D.T., Marion, S., Griffiths, G., Watanabe, T., Kaibuchi, K. and Grosse, R. (2007) Dia1 and IQGAP1 Interact in Cell Migration and Phagocytic Cup Formation. The Journal of Cell Biology, 178, 193-200. http://dx.doi.org/10.1083/jcb.200612071

[38] Naj, X., Hoffmann, A.K., Himmel, M. and Linder, S. (2013) The Formins FMNL1 and mDia1 Regulate Coiling Phagocytosis of Borrelia burgdorferi by Primary Human Macrophages. Infection and Immunity, 81, 1683-1695. http://dx.doi.org/10.1128/IAI.01411-12

[39] Van Goethem, E., Guiet, R., Balor, S., Charriere, G.M., Poincloux, R., Labrousse, A., Maridonneau-Parini, I. and Le Cabec, V. (2011) Macrophage Podosomes Go 3D. European Journal of Cell Biology, 90, 224-236. http://dx.doi.org/10.1016/j.ejcb.2010.07.011

[40] Seth, A., Otomo, C. and Rosen, M.K. (2006) Autoinhibition Regulates Cellular Localization and Actin Assembly Activity of the Diaphanous-Related Formins FRLalpha and mDia1. The Journal of Cell Biology, 174, 701-713. http://dx.doi.org/10.1083/jcb.200605006 